\title{
Design of central air conditioning controller with remote control based on STM32
}

\author{
Qiu Gao ${ }^{\mathrm{a}}$, Yan Dong ${ }^{\mathrm{b}}$, Liang-Wen Yan ${ }^{\mathrm{c},}{ }^{\dagger}$, Xue Yu ${ }^{\mathrm{d}}$ and Nian-Feng Mei ${ }^{\mathrm{e}}$ \\ ${ }^{1}$ Room 419, Mechanical building, 149 Yanchang Road, Zhabei District, \\ Shanghai, China \\ ${ }^{a}$ gaoqiu1990@163.com, ${ }^{b} u j n \_j z d y @ 126 . c o m,{ }^{c} L w \_y a n @ s t a f f . s h u . e d u . c n$ \\ d522129793@qq.com, ${ }^{e} 1069164172 @ q q . c o m$
}

\begin{abstract}
At present, the decentralized control was adopted at the terminal of the central air conditioning system so that cluster management couldn't be achieved. Taking into account such as different user had different management level and unreasonable, unscientific control methods which leaded to great waste of energy. In order to solve this problem to a certain extent, this paper described a kind of central air conditioning controller with remote control based on STM32. The design not only provided a set of solution schema for its cluster control, but also achieved the remote control and remote locking of the central air-conditioning, as a result of which it extended the service life of the central air conditioning device, and reduced the energy consumption of central air conditioning. Through the design, energy-saving purposes had been reached.
\end{abstract}

Keywords: STM32; Central air conditioning; Remote control; Energy saving; Cluster control.

\section{Introduction}

Central air conditioning occupies a major part of building energy consumption. With the promotion of the national policy, energy saving and emission reduction, the central air-conditioning as the main energy saving research object has aroused widespread concern within the industry. At present, at terminal of the central air conditioning system, the decentralized control is adopted; Cluster management can't be achieved. Taking into account such as different user had different management level and unreasonable, unscientific control methods, which leaded to great waste of energy. Now many high-grade hotel lobbies have installed central air-conditioning terminal controller without remote control, in order to display the current temperature and adjust the temperature of the lobby. However, body feeling of different people corresponding to apparent temperature is different. Some people who feel hot for a certain temperature will go to adjust the thermostat to lower the temperature. At this time, central air-conditioning terminal controller will control central air-conditioning fan coil and the electromagnetic valve full load work to meet the user's needs. But other 
people may think that the temperature is too low and feel cold, and then to adjust the thermostat again. In the similar cases as being so frequently adjusted, the central air-conditioning fan switches in the high, the medium and the low speed and is always under a full speed running state .Meanwhile, a large amount of energy will be consumed, which is not desirable.

Although there are a lot of central air-conditioning controllers with remote control in sale, the main concern of these controllers is to monitor remotely, set relevant parameters of the central air-conditioning controller and use RS485 or Zigbee to achieve cluster control of the central air-conditioning, which does not solve the increase of energy consumption, caused by users' operation frequently.

With the continuous development of science, information technology and microprocessor technology, the realization of energy-saving strategy of central air conditioning has become possible. This paper described a kind of the central air conditioning terminal controller with remote control based on STM32 (Cortex_M3 kernel), which was used to achieve energy-saving of central air conditioning. On the one hand, it provided the possibility to realize the Cluster control of central air conditioning system. On the other hand, it provided a solution for the energy-saving of central air conditioning system. To prevent the central air conditioning frequently operated by different people and save energy, the host computer or mobile terminal, such as mobile phone, can control the central air conditioning through the central air conditioning controller, and lock central air conditioning controller, which ensures that the central air conditioning works at a suitable and fixed temperature as far as possible. In order to meet various people's needs for comfortable feeling, the author had counted temperature set by people on the terminal controller of central air conditioner in the PC software, then got the most suitable temperature in different seasons of the year by data mining algorithm, and made these temperatures as reference values of remote setting temperature. Of course, control personnel can set an appropriate temperature based on experience.

\section{System Overall Scheme Design}

The terminal controller of central air conditioning system is composed of two parts: hardware system and software system.

Hardware system includes power module, CPU (ARM microcontroller), temperature detection module, LCD driver module, LCD monitor, RS485 communication module, function keys and watchdog module, etc. The system structure diagram is shown in Figure 1. 


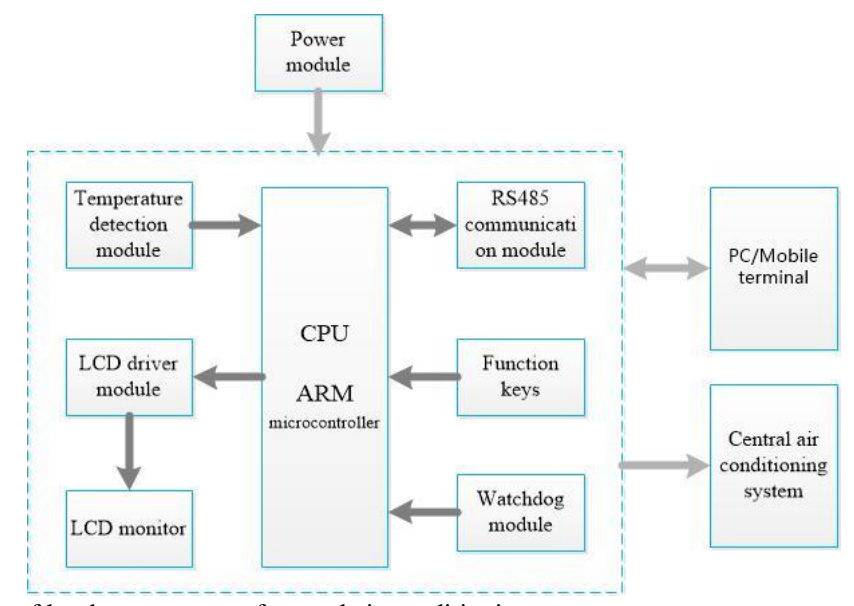

Fig.1. Structure of hardware system of central air conditioning system

System working principle: Power module provides the necessary working power for CPU, temperature sensor, LCD driver module, LCD, RS485 communication module and the watchdog module. CPU collects temperature detected by the temperature sensor, and drivers LCD to display the temperature. The user can set the required temperature by the function button, and the temperature value is displayed on the LCD by the CPU processing. Users can also use the function button to set the working mode and wind speed. After a temperature value has been set, CPU will compare it with the temperature value detected by the temperature sensor. Based on the results of the comparison, CPU will control central air conditioning work through the central air-conditioning control interface. The watchdog module is used to ensure the normal operation of the embedded application in the central air conditioning terminal controller. Beside this, it can also resume the application and can make the application in normal working condition again when the embedded application program runs out for any reason. PC software communicates with the central air-conditioning controller through RS485 communication module. The set temperature, wind speed and central air-conditioning operation mode setting, display of temperature detected by temperature sensor as well as central air conditioning controller remote locking can be completed on the PC software. The central air conditioning controller remote lock is the characteristic function of the controller. It is to avoid the central air conditioning controller frequently being operated and make the central air conditioning related device life decline and central air-conditioning energy consumption increase, so as to achieve the aim of saving energy. 
Software system includes embedded application software based on $\mathrm{C}$ language, $\mu \mathrm{C} / \mathrm{OS} \_$II embedded real-time operating system, MODBUS communication protocol stack and PC software.

\section{Hardware System Design}

\subsection{A central processing unit (CPU)}

With the development of the microprocessor (MCU) technology, a variety of high-performance, high speed, high reliability, high cost performance microprocessors are brought out, for example, Atmel's 51 series, AVR MCU, ARM microprocessor; ST's STM8 and STM32 series of microprocessors. However, STM32 series microprocessors manufactured by ST company have an excellent prospect with high cost performance, high processing speed, improved the development environment and tools, rich abundance of reference material.

After the comprehensive consideration, STM32F103C8T6 chip is most appropriate choice as the main control chip. In order to ensure that the STM32F103C8T6 chip and related peripheral equipment work properly, the minimum device and circuit must be designed to ensure the normal operation of the system. This is called the STM32F103C8T6 minimum system and the schematic diagram shown in Figure 2.

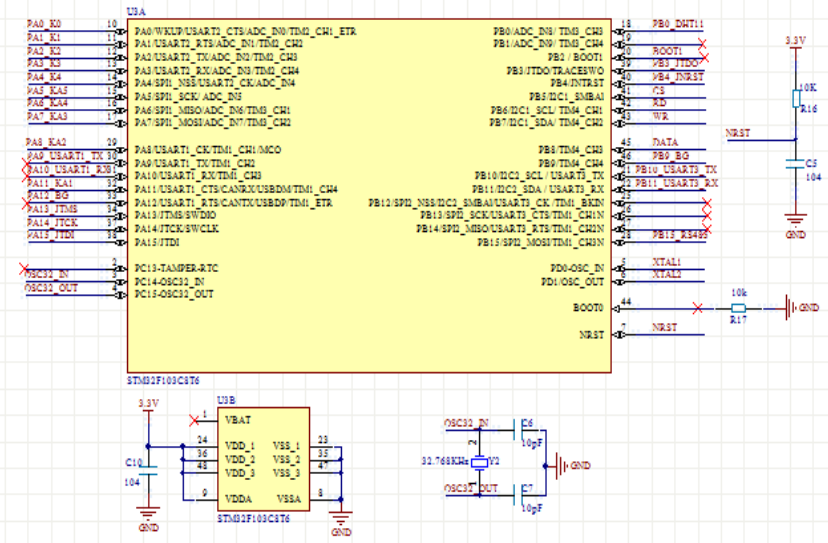

Fig. 2 STM32F103C8T6 minimum system schematic diagram

\subsection{Power module}

The power module provides the power supply for each module of the terminal controller. Two kinds of the power are required over the entire central air-conditioning controller. The power of $3.3 \mathrm{~V}$ is supplied for the CPU, temperature sensor, LCD and RS485 communication IC. The power of $5.0 \mathrm{~V}$ is 
supplied for the LCD driver IC. Schematic of the power module is shown in Figure 3.

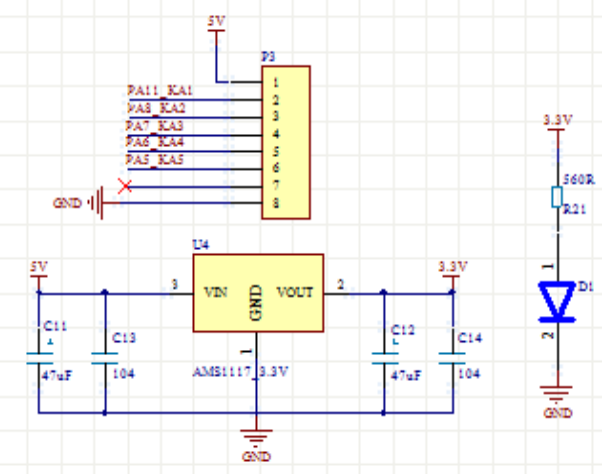

Fig. 3 Schematic diagram of power module

\subsection{LCD Driver module and LCD display}

The LCD driver module is used to drive the LCD display the detected temperature, the set temperature, the working mode and the wind speed. Currently, there are a wide variety of displays available on the market, such as TFT LCD, OLED LCD and Segment LCD, etc. By comparison, segment LCD can meet the needs of the central air conditioning controller, the cost performance of which is higher than the TFT LCD and OLED LCD. In the design schema, four-segment LCD is chosen as the display of central air conditioning controller. Its schematic diagram is shown in Figure 4.
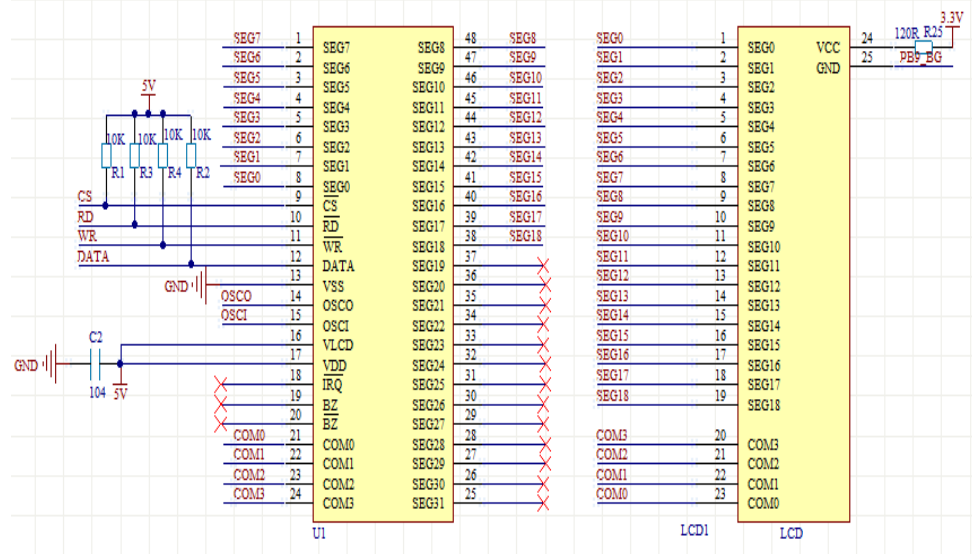

HT1621B

Fig. 4 Schematic diagram of LCD driver and LCD display 


\subsection{RS485 communication module}

In order to achieve the remote control and the lock, central air conditioning controller must be able to communicate with the host computer. The controller uses Maxim produced a cost-effective and sophisticated communications IC MAX485. On the basis of this, The Modbus communication protocol stack of our own writing is adopted to realize the communication between the central air conditioning controller and the host computer. Its schematic diagram is shown in Figure 5 .

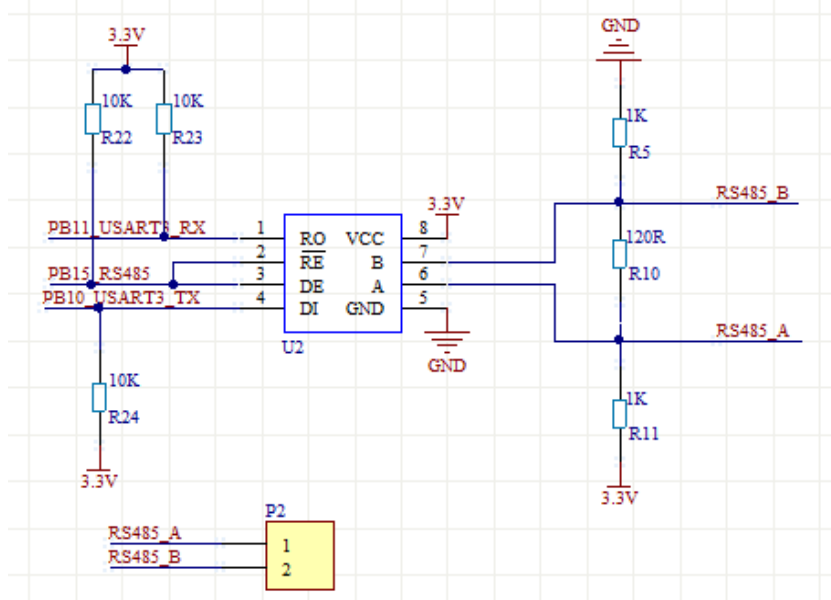

Fig. 5 Schematic diagram of RS485 communication module

\subsection{Function keys}

Function keys are important devices to achieve human-computer interaction (HCI). The setting of the central air conditioning controller can be realized through the function keys, including the temperature setting, the working mode and the wind speed of the wind turbine setting. Its schematic diagram is shown in Figure 6. 


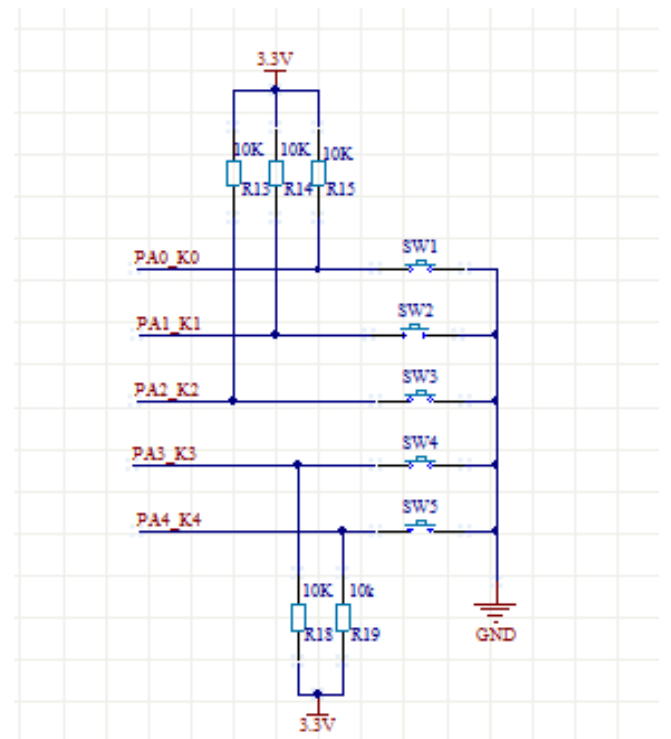

Fig. 6 Schematic diagram of the function keys

\subsection{Temperature detection module}

The temperature detection module is used to detect the indoor temperature. An industrial grade temperature sensor DS18B20 is adopted in the temperature detection module. DS18B20 temperature sensor is a high precision single bus temperature sensor produced by American DALLAS company. It has better performance and price performance. Its schematic diagram is shown in Figure 7.

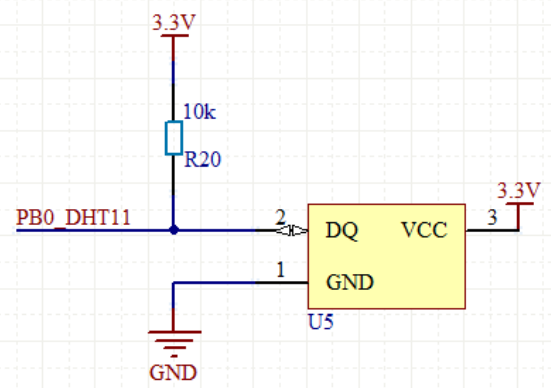

Fig. 7 schematic diagram of temperature detection module 


\section{Software System Design}

\subsection{Software architecture}

To achieve the above functions, the software design aspect, $\mu \mathrm{C} / \mathrm{OS}$ II embedded real-time operating system of the excellent performance is used. It can not only improve the efficiency of the CPU, but also realize real-time communication which "bare-metal program" can't do. After using the operating system can guarantee real-time and stability of communication, this is what all communication equipment must be guaranteed. In the software system, three threads are used to complete all of the above functions. Thread key_task ( ) is used to process key information, thread Lcd_task ( ) to process segment LCD display, and thread Modbus_task ( ) to process communication. The overall design framework of software system is shown in Figure 8.

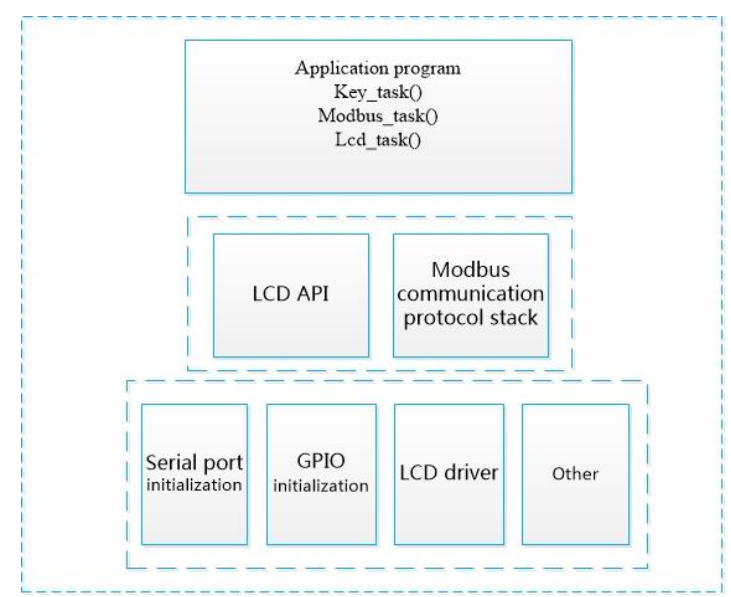

Fig. 8 block diagram of software system

At the bottom of the software system is the hardware initialization program. GPIO initialization procedure is used to initialize all the I/O ports used. Serial port initialization procedure is used to initialize the port of communication. It is the basis for the work of the Modbus communication protocol stack. LCD driver is used to initialize the segment LCD and provide API function of segment LCD display.

In the middle layer of the software system is the application program interface. LCD API function is written by invoking to LCD driver API functions of the hardware layer. Modbus communication protocol stack is a full function Modbus communication protocol based on $\mathrm{C}$ language. It is the core software of the central air conditioning controller and the host computer communication. 
At the top of the software system is the application that is used to achieve the desired functionality of the user. It completes the task by calling the API function provided by the middle layer of the software system.

\subsection{Tware work flow}

The process of central air-conditioning controller software executed as shown in Figure 9.

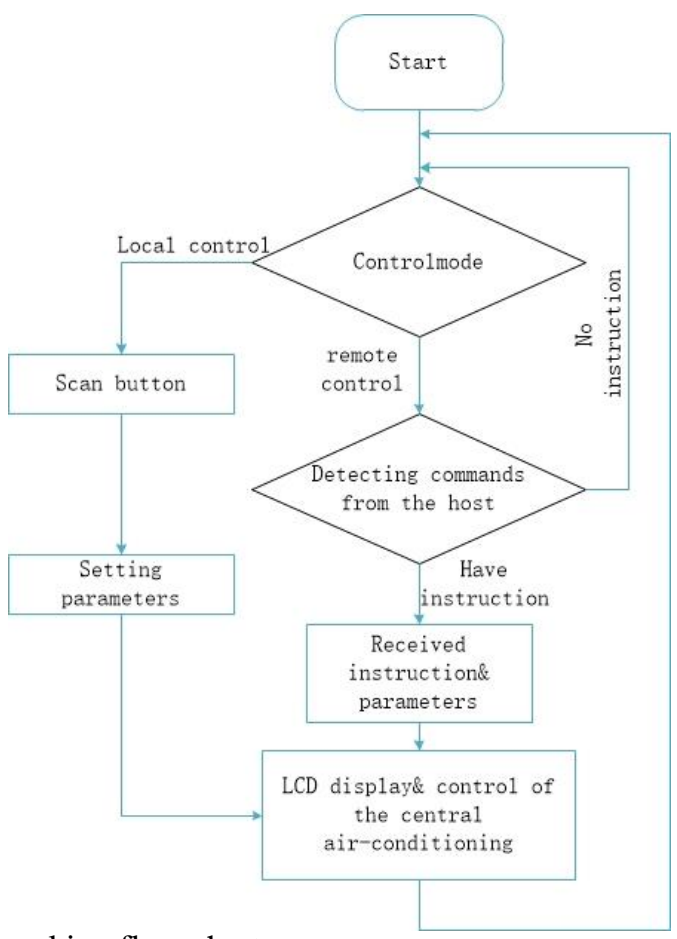

Fig. 9 software working flow chart

\subsection{Remote controller lock}

In embedded applications, a global variable is defined. The user can set the value of the global variable applied by the host computer software. The embedded application software can detect and analyze the value of the variable in real time. If the value of the variable satisfies the remote control condition, the embedded application will switch to the remote control part. In this case, the controller is locked and user actions on the controller are invalid. This reduces the power consumption of the central air conditioner brought by the users' frequent operations of the thermostat. 


\section{Summary}

This paper describes the design of the terminal controller of the central air conditioning system based on STM32 with remote control. The design of hardware and software system is described in details, including STM32 minimum system, power module, RS485 communication module, LCD driver module and watchdog module. The design not only provided a set of solution schema for its cluster control, but also achieved the remote control and remote locking of the central air-conditioning, as a result of which it extended the service life of the central air conditioning device, and reduced the energy consumption of central air conditioning. Through the design, energy-saving purposes had been reached. This design not only has a great significance on achieving low carbon life, but also is a best response to energy saving response.

\section{References}

[1]. Yujian Liu, Quanlei Yin, Pengfei Zheng. Temperature Controller of Central Air Condition Based on STC MCU [J]. Programmable Controller Factory Automation (FA PLC), 2011 (3): 100-101. (In Chinese)

[2]. LiFei Wu, Feng Ye, Zhangyan Yang, etc. The central air-conditioning intelligent terminal thermostat based on Zigbee [J]. Machinery Design \& Manufacture, 2013 (3): 74-76. (In Chinese)

[3]. Yanyang Zhang. Study on Monitoring System for HVAC Thermostat Controller Based on Zig Bee [D]. South China University of Technology, 2014. (In Chinese)

[4]. Zhe Ren, hongzheng Fang, Jing Cao. Embedded real time operating system $\mu \mathrm{C} / \mathrm{OS}-\mathrm{II}$ principle and application (Third Edition) [M] (3rd edition) Beijing: Beijing University of Aeronautics and Astronautics Press .2014.1. (In Chinese)

[5]. Texas Instrument s. STM32F104 Datasheet [R]. 2007.

[6]. Brian W. Kernighan \& Dennis M. Ritchie. C Programming Language [M] (Second Edition) Baowen $\mathrm{Xu}$, Zhiyi Li translated. Beijing: Mechanical Industry Press .2004.1. (In Chinese)

[7]. Bowen Liu, Yan Sun. Embedded real time operating system $\mu \mathrm{C} / \mathrm{OS}$ _II classic example - based on STM32 processor [M] (2nd edition) Beijing: Beijing University of Aeronautics and Astronautics Press .2014.5. (In Chinese)

[8]. Jun Liu, Yang Zhang, HanYu Yan. For example STM32 [M] (Second Edition). Beijing: Beijing University of Aeronautics and Astronautics Press.2014.6. (In Chinese) 